\title{
Transdermal Electrical Stimulation in Sensorineural Tinnitus
}

S Maini, Senior Resident, B J Medical College, Pune - 411001

S C Deogaonkar Associate Professor, Departments of ENT. Government Medical College,

Chandigarh. Pin 16004

\section{||||||||||||||||||||||||||||||||||||||||||||||||||||||||||||||||||||||||||||||||||||||||||||||||||||||||||||||||||}

\section{Abstract}

In 73 patients composing 84 ears, with persistent decompensated tinnitus and sensorineural deafness, tinnitus suppression was attempted with low frequency, low ampere transdermal electrical stimulation (TDES). The causes of tinnitus were presbyacusis, unknown aetiology, Noise exposure, otosclerosis, ototoxic drugs, Endolymphatic hydrops, labyrinthitis and post head injury. Reduction of tinnitus was achieved in 38 of 84 ears. Results were achieved in patients with the following order. 1) Unknown aetiology 53.3\% 2) Ototoxic drugs 50\% 3) Presbyacusis 45\% 4) Labyrinthitis $40 \%$ 5) Post head injury, Endolymphatic hydrops \& otosclerosis $33.3 \%$ each. The lasting effect of the tinnitus suppression varied from few hours to few weeks. Effective reduction could be re-achieved by re-stimulation.

\section{||||||||||||||||||||||||||||||||||||||||||||||||||||||||||||||||||||||||||||||||||||||||||||||$+|||||||||||||||||| \mid$}

\section{Introduction}

Deports of much reduced or abolished tinnitus by electrical stimulation with cochlear implants (House 1970) created a revived interest in treatment of tinnitus by electrical stimulation. Subsequently electrical stimulation for Tinnitus suppression has been attempted by stimulation of various sites such as Tympanic membrane, promontory, mastpod bone, round window \& scala tympani with variable success rates of Tinnitus suppression ranging from 20$85 \%$. (Vernon et al 1985, Kaada et al 1989, Techinbana et al 1992). Most studies reveal its effectiveness in cochlear lesions. Thus electrical stimulation may be effective for tinnitus with inner ear lesions. However there are only few studies concerning this problem, therefore a study to evaluate the effectiveness of External electrical stimulation, in Tinnitus, in sensorineural deaf ears was taken up.

\section{Materials and Methods}

Seventy three patients with either unilateral or bilateral intractable tinnitus and sensorineural hearing loss who visited Sássoon General Hospital from 1990 to 1993 were selected for the study. The duration of the symptoms varied from one month to 20 years. Age range of patients was $14-80$ years. Each patient was examined as follows: 1 . Thorough History 2 . Complete Medical Examination 3. Detailed ENT examination 4. Pre \& post stimulation audiological assessment 5. Haemogram, hearing tests, caloric test, radiological assessment \& haematologic investigation as indicated. The clinical diagnosis of the patients and the number of ears involved were as follows: Presbyacusis 40. Unknown cause 15, Noise induced hearing loss 11, labyrinthitis 5, ototoxic drugs 4, Endolymphatic hydrops 3, Post head injury 3 , otosclerosis 3. TDES was 
given with cutaneous electrodes. The cathode was placed postaurally on the wet skin surface of the mastoid process of the affected ear, while the anode was the indifferent electrode placed on forehead/neck. Positive pulses of $0.01 \mathrm{msec}$ duration at a rate of $100 \mathrm{~Hz}$ were selected and starting from $10 \mathrm{v}$ was gradually increased till the level of pain sensation, usually ranging between 20-40 volts. Stimulation was given for 4 successive days lasting 15 minutes each. The decrease in tinnitus was subjectively evaluated during and $f$ llowing each stimulation. Patients with decrease in tinnitus, following first 4 stimulations were further stimulated twice a week for $5-6$ weeks. Patients with significant relief i.e. subjective tinnitus getting compensated for a few days atleast were taken as successes. Patients with no relief following the first 4 stimulations were not subjected to further stimulations.

\section{Results}

\section{Effectiveness}

Reduction of Tinnitus was observed in 38 out of 84 sensorineural deaf ears $(40.4 \%)$. Table 1 shows the number of cases and the rate of Tinnitus suppression for each disease. TDES was most effective in case of tinnitus of unknown etiology $8 / 15(53.3 \%)$ followed by ototoxicity $2 / 4(50 \%)$, Noise induced hearing loss 5/11 (45.4\%), Presbyacusis 18/40 (45\%),

Table 1. Tinnitus suppression by TDES for differet etiologies

\begin{tabular}{lrc}
\hline Tinnitus & \multicolumn{2}{c}{ Suppression } \\
\hline Probable cause & \multicolumn{1}{c}{ Ears } & Percentage \\
\hline Presbyacusis & $18 / 40$ & 45.0 \\
Unknown aetiology & $8 / 15$ & 53.3 \\
Noise & $5 / 11$ & 45.4 \\
Labyrinthitis & $2 / 5$ & 40.0 \\
Ototoxic drugs & $2 / 4$ & 50.0 \\
Post head injury & $1 / 3$ & 33.3 \\
Endolymphatic hydrops $1 / 3$ & 33.3 \\
Otosclerosis & $1 / 3$ & 33.3 \\
\hline Total & $38 / 84$ & 45.2 \\
\hline
\end{tabular}

labyrinthitis $2 / 5(40 \%)$, post head injury $1 / 3$ $(33.3 \%)$, Endolymphatic hydrops $1 / 3(33.3 \%)$ and otosclerosis $1 / 3(33.3 \%)$.

\section{Duration of Tinnitus Suppression}

38 patients had tinnitus suppression following TDES. 6 ears had complete suppression of Tinnitus in 6 months follow up. 32 Patients had partial suppression with conversion of decompensated tinnitus into compensated tinnitus with lasting effect varying between few days to few weeks. On recurrence of troublesome tinnitus, the beneficial effects of TDES could be re-obtained by re-stimulation similarly.

\section{Relationship between duration of Tinnitus and hearing level changes and Tinnitus Sup- pression.}

No relationship was established between the duration of syndrome and tinnitus suppression and no significant hearing level changes were observed in post-stimulation pure tone Audiometry.

\section{Side-Effects}

TDES is a safe procedure. Only 6 patients had heaviness in the head, 3 had slight temporary aggravation of Tinnitus and 4 had mild giddiness following stimulation.

\section{Discussion}

Even though many modes of action of TDES are postulated such as increased Micro-circulation due to sympatho-inhibition, central- inhibition, stimulation of cutaneous nerve fibres, the exact mechanism is not known. Engelberg et al evaluated TDES to treat tinitus in an experimental study. A single blind protocol with 20 subjects comprising 33 ears with tinnitus was carried out. $82 \%$ of the 33 ears showed improvement. Rostovtsev et al used TDES in 62 patients suffering from tinnitus. Complete disappearance of tinnitus in 20 patients $(32.3 \%)$ and partial tinnitus suppression in 25 patients $(40.3 \%)$ was achieved. In the present study 78 ears with tinnitus and sensorineural deafness 
received TDES and 38 ears had considerable relief of tinnitus. The wide range of results reported in studies by various investigators, may be due to improper identification of clinical types of tinnitus. Most studies found its maximum efficacy in cochlear type of tinnitus.
TDES is now being considered as a viable mode to Tinnitus control. Commercial devices are under trial using the principal of TDES for Tinnitus suppression as a non traumatic, simple procedure, easily done in the out patient clinic.

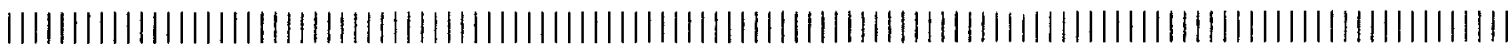

\section{References}

1. Engelberg M. Bauer W: (1985) Transcutaneous electrical stimulation for tinnitus, laryngoscope. 95 (10): 1167-73.

2. House WF. 11976). Cochlear implants. Ann Otol Rhinol Laryngol suppl. 185: 1-93.

3. Kaada B, Hognestad, Havstad I: (1985) Transcutaneous nerve stimulation in tinnitus report of progress. Acta Otorhinolaryngol. Belg 39 (3): 621-37.

4. Rostovtsev VN, Kofanov RV: (1992) Indirect electric stimulation of the auditory neural structures in the complex treatment of sensorineural hearing loss and subjective noise in the ears. Vestn Oto Rhino langngo. 3:13-4.

5. Tachibana M, Kiyoshitay, Senuma H. Nikanishi H, Sasaki K. (1992). Effect of transcutaneous electrostimulation on noise induced temporary threshold shift. Acts otolaryna (Stockh) ; 112 (4): 595-8.

6. Vernon JA: (1985) Research in tinitus report of progress. Acts. Otorhinolaryngol. Belg; 39 (3): $621-37$. 